\title{
Crystal structure of methyl ferrocenemonocarboxylate, $\left[\mathrm{Fe}\left(\mathrm{C}_{5} \mathrm{H}_{5}\right)\left(\mathrm{C}_{5} \mathrm{H}_{4} \mathrm{C}_{2} \mathrm{O}_{2} \mathrm{H}_{3}\right)\right]$
}

\author{
W. Beck*, O. E. Woisetschläger and P. Mayer
}

Ludwig-Maximilians-University, Department of Chemistry, Butenandtstraße 5-13 (D), D-81377 Munich, Germany

Received February 13, 2001, CCDC-No. 1267/619

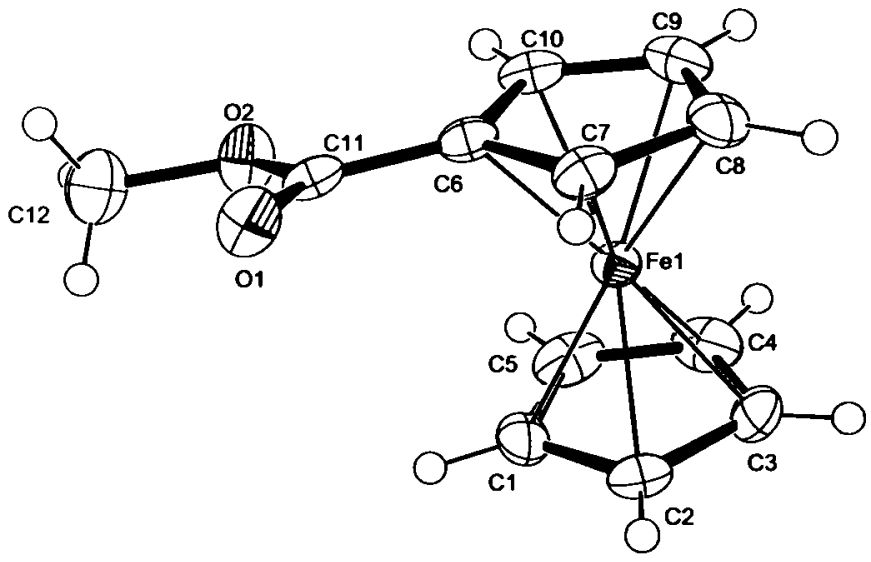

Table 1. Data collection and handling.

Crystal:

Wavelength:

$\mu$ :

Diffractometer, scan mode:

$2 \theta_{\max }:$

$N(h k l)_{\text {measured, }} N(h k l)_{\text {unique: }}$

Criterion for $I_{\text {obs, }} N(h k l)_{\text {gt }}$ :

$N(\text { param })_{\text {Tefined: }}$

Programs:

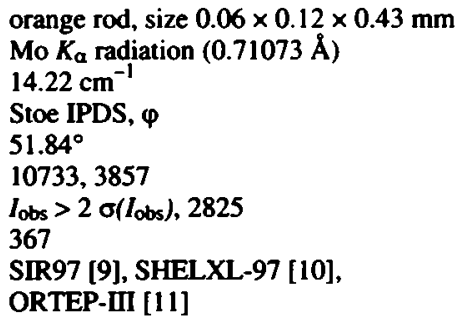

Table 2. Atomic coordinates and displacement parameters (in $\AA^{2}$ ).

\begin{tabular}{llllll}
\hline Atom & Site & $\boldsymbol{x}$ & \multicolumn{1}{l}{$\boldsymbol{y}$} & \multicolumn{1}{l}{$U_{\text {iso }}$} \\
\hline H(1) & $4 e$ & $0.814(2)$ & $0.224(5)$ & $0.740(1)$ & $0.045(8)$ \\
H(2) & $4 e$ & $0.857(2)$ & $-0.144(5)$ & $0.791(1)$ & $0.031(7)$ \\
H(3) & $4 e$ & $0.841(2)$ & $-0.126(5)$ & $0.895(1)$ & $0.036(8)$ \\
H(4) & $4 e$ & $0.792(2)$ & $0.257(5)$ & $0.912(1)$ & $0.043(8)$ \\
H(5) & $4 e$ & $0.776(2)$ & $0.479(5)$ & $0.817(1)$ & $0.036(8)$ \\
H(7) & $4 e$ & $0.646(2)$ & $-0.297(5)$ & $0.730(1)$ & $0.032(7)$ \\
H(8) & $4 e$ & $0.644(2)$ & $-0.312(5)$ & $0.837(1)$ & $0.043(8)$ \\
H(9) & $4 e$ & $0.593(2)$ & $0.069(5)$ & $0.861(1)$ & $0.035(7)$ \\
H(10) & $4 e$ & $0.570(2)$ & $0.326(5)$ & $0.771(1)$ & $0.022(7)$ \\
H(12A) & $4 e$ & $0.586(2)$ & $0.601(7)$ & $0.604(2)$ & $0.06(1)$ \\
H(12B) & $4 e$ & $0.534(2)$ & $0.380(6)$ & $0.569(2)$ & $0.058(9)$ \\
H(12C) & $4 e$ & $0.637(2)$ & $0.410(5)$ & $0.592(1)$ & $0.026(7)$ \\
H(13) & $4 e$ & $0.799(2)$ & $-0.463(7)$ & $0.670(2)$ & $0.08(1)$ \\
H(14) & $4 e$ & $0.969(2)$ & $-0.466(7)$ & $0.700(2)$ & $0.07(1)$ \\
H(15) & $4 e$ & $1.015(2)$ & $-0.079(5)$ & $0.679(1)$ & $0.046(8)$ \\
H(16) & $4 e$ & $0.885(2)$ & $0.156(6)$ & $0.638(1)$ & $0.040(9)$ \\
H(17) & $4 e$ & $0.757(2)$ & $-0.084(6)$ & $0.635(1)$ & $0.051(9)$ \\
H(19) & $4 e$ & $0.802(2)$ & $-0.626(5)$ & $0.532(1)$ & $0.027(7)$ \\
H(20) & $4 e$ & $0.964(2)$ & $-0.615(5)$ & $0.567(1)$ & $0.028(7)$ \\
H(21) & $4 e$ & $1.010(2)$ & $-0.223(5)$ & $0.545(1)$ & $0.029(7)$ \\
H(22) & $4 e$ & $0.872(1)$ & $0.007(5)$ & $0.503(1)$ & $0.022(7)$ \\
H(24A) & $4 e$ & $0.608(2)$ & $0.241(6)$ & $0.467(1)$ & $0.051(9)$ \\
H(24B) & $4 e$ & $0.579(2)$ & $0.030(6)$ & $0.498(2)$ & $0.07(1)$ \\
H(24C) & $4 e$ & $0.575(2)$ & $0.019(5)$ & $0.428(1)$ & $0.039(8)$ \\
\hline & & & & & \\
\hline
\end{tabular}

\begin{abstract}
$\mathrm{C}_{12} \mathrm{H}_{12} \mathrm{FeO}_{2}$, monoclinic, $P 12_{1} / c 1$ (No. 14), $a=16.064(1) \AA$, $b=5.9250(3) \AA, c=23.105(2) \AA, \beta=108.79(1)^{\circ}, V=2081.9 \AA^{3}$, $Z=8, R_{\mathrm{gt}}(F)=0.029, w R_{\text {ref }}\left(F^{2}\right)=0.053, T=200 \mathrm{~K}$.
\end{abstract}

\section{Source of material}

The title complex [1] was synthesized from ferrocenecarboxylic acid and thionylchloride in methanol and extracted with $\mathrm{n}$-pentane. Crystals were obtained by sublimation in vacuum.

\section{Discussion}

Ferrocenecarboxylic acid esters are well studied compounds [2] and can be used, e.g., for the synthesis of ferrocenyl substituted 1,3-diketones [3-5]. The crystallographic data of ethyl and phenyl ferrocenecarboxylate were reported [6]. In the title molecule the ferrocene unit deviates from the eclipsed conformation by $\delta=$ $4.6^{\circ}$. The $\mathrm{C} 6-\mathrm{Cl} 1$ bond distance between the $\mathrm{Cp}$ ring and the carboxyl group is with $1.464(3) \AA$ shorter than a typical C-C single bond which corresponds to somewhat longer $\mathrm{C} 6-\mathrm{C} 7$ and C6 $-\mathrm{C} 10$ bond lengths $(1.43 \AA)$ in the $\mathrm{Cp}$ ring compared to $\mathrm{C} 7-\mathrm{C} 8, \mathrm{C} 8-\mathrm{C} 9$ and $\mathrm{C} 9-\mathrm{C} 10(1.42 \AA)$. All the bond lengths (C6- $\mathrm{C} 11, \mathrm{C}-\mathrm{C}_{\text {ring, }} \mathrm{C}=\mathrm{O}, \mathrm{C}-\mathrm{O}$ ) are quite similar to that of ferrocenecarboxylic acid [7] and of ferrocene-1,1'-dicarboxylic acid [8]. The ester unit is coplanar with the $\mathrm{C}_{5} \mathrm{H}_{4}$ ring

\footnotetext{
* Correspondence author (e-mail: wbe@cup.uni-muenchen.de)
} 
Table 3. Atomic coordinates and displacement parameters (in $\AA^{2}$ ).

\begin{tabular}{|c|c|c|c|c|c|c|c|c|c|c|}
\hline Atom & Site & $x$ & $y$ & $z$ & $U_{11}$ & $U_{22}$ & $U_{33}$ & $U_{12}$ & $U_{13}$ & $U_{23}$ \\
\hline $\mathrm{Fe}(1)$ & $4 e$ & $0.71287(2)$ & $0.06234(6)$ & $0.80378(1)$ & $0.0185(2)$ & $0.0228(2)$ & $0.0197(2)$ & $-0.0030(2)$ & $0.0044(1)$ & $-0.0035(2)$ \\
\hline $\mathrm{Fe}(2)$ & $4 e$ & $0.88075(2)$ & $-0.26311(6)$ & $0.59627(1)$ & $0.0227(2)$ & $0.0238(2)$ & $0.0147(1)$ & $0.0044(1)$ & $0.0052(1)$ & $-0.0005(2)$ \\
\hline$O(1)$ & $4 e$ & $0.6054(1)$ & $0.0045(3)$ & $0.62554(7)$ & $0.0384(9)$ & $0.026(1)$ & $0.0287(9)$ & $0.0041(7)$ & $0.0057(7)$ & $-0.0056(8)$ \\
\hline$O(2)$ & $4 e$ & $0.5940(1)$ & $0.3519(3)$ & $0.66031(8)$ & $0.048(1)$ & $0.022(1)$ & $0.030(1)$ & $0.0035(8)$ & $0.0097(8)$ & $-0.0012(8)$ \\
\hline$O(3)$ & $4 e$ & $0.6482(1)$ & $-0.3544(3)$ & $0.48333(9)$ & $0.0296(9)$ & $0.026(1)$ & $0.052(1)$ & $-0.0068(8)$ & $0.0053(8)$ & $0.0005(9)$ \\
\hline$O(4)$ & $4 e$ & $0.6963(1)$ & $-0.0006(3)$ & $0.47972(7)$ & $0.0247(8)$ & $0.023(1)$ & $0.0378(9)$ & $0.0002(7)$ & $0.0028(7)$ & $0.0083(8)$ \\
\hline$C(1)$ & $4 e$ & $0.8153(2)$ & $0.1959(5)$ & $0.7813(1)$ & $0.023(1)$ & $0.042(2)$ & $0.029(1)$ & $-0.010(1)$ & $0.007(1)$ & $0.005(1)$ \\
\hline$C(2)$ & $4 e$ & $0.8391(1)$ & $-0.0176(5)$ & $0.8086(1)$ & $0.018(1)$ & $0.034(2)$ & $0.038(1)$ & $0.001(1)$ & $0.007(1)$ & $-0.003(1)$ \\
\hline$C(3)$ & $4 e$ & $0.8312(2)$ & $-0.0091(5)$ & $0.8677(1)$ & $0.023(1)$ & $0.048(2)$ & $0.027(1)$ & $-0.001(1)$ & $-0.001(1)$ & $0.009(1)$ \\
\hline$C(4)$ & $4 e$ & $0.8032(2)$ & $0.2094(6)$ & $0.8770(1)$ & $0.028(1)$ & $0.061(2)$ & $0.026(1)$ & $-0.013(1)$ & $0.004(1)$ & $-0.020(2)$ \\
\hline$C(5)$ & $4 e$ & $0.7930(2)$ & $0.3366(6)$ & $0.8234(1)$ & $0.026(1)$ & $0.022(2)$ & $0.058(2)$ & $-0.006(1)$ & $0.004(1)$ & $-0.006(1)$ \\
\hline$C(6)$ & $4 e$ & $0.6049(1)$ & $0.0508(5)$ & $0.7273(1)$ & $0.016(1)$ & $0.023(2)$ & $0.028(1)$ & $-0.002(1)$ & $0.0034(9)$ & $-0.006(1)$ \\
\hline$C(7)$ & $4 e$ & $0.6289(1)$ & $-0.1726(5)$ & $0.7504(1)$ & $0.021(1)$ & $0.024(2)$ & $0.030(1)$ & $-0.004(1)$ & $0.003(1)$ & $-0.005(1)$ \\
\hline$C(8)$ & $4 e$ & $0.6252(2)$ & $-0.1801(5)$ & $0.8109(1)$ & $0.025(1)$ & $0.034(2)$ & $0.034(1)$ & $-0.011(1)$ & $0.010(1)$ & $-0.000(1)$ \\
\hline $\mathrm{C}(9)$ & $4 e$ & $0.5989(2)$ & $0.0355(5)$ & $0.8252(1)$ & $0.026(1)$ & $0.042(2)$ & $0.035(1)$ & $-0.007(1)$ & $0.016(1)$ & $-0.009(1)$ \\
\hline$C(10)$ & $4 e$ & $0.5867(1)$ & $0.1792(5)$ & $0.7742(1)$ & $0.017(1)$ & $0.028(2)$ & $0.039(1)$ & $0.002(1)$ & $0.007(1)$ & $-0.008(1)$ \\
\hline$C(11)$ & $4 e$ & $0.6022(1)$ & $0.1275(4)$ & $0.6665(1)$ & $0.014(1)$ & $0.025(2)$ & $0.030(1)$ & $0.0008(9)$ & $0.0007(9)$ & $-0.004(1)$ \\
\hline$C(12)$ & $4 e$ & $0.5863(2)$ & $0.4397(7)$ & $0.6003(1)$ & $0.052(2)$ & $0.029(2)$ & $0.037(2)$ & $0.001(2)$ & $0.010(1)$ & $0.002(1)$ \\
\hline$C(13)$ & $4 e$ & $0.8397(2)$ & $-0.3375(6)$ & $0.6687(1)$ & $0.075(2)$ & $0.053(2)$ & $0.028(2)$ & $-0.008(2)$ & $0.029(2)$ & $0.001(1)$ \\
\hline$C(14)$ & $4 e$ & $0.9313(2)$ & $-0.3381(7)$ & $0.6868(1)$ & $0.076(2)$ & $0.055(2)$ & $0.016(1)$ & $0.030(2)$ & $0.006(1)$ & $0.005(1)$ \\
\hline$C(15)$ & $4 e$ & $0.9596(2)$ & $-0.1258(7)$ & $0.6755(1)$ & $0.032(2)$ & $0.075(3)$ & $0.025(1)$ & $-0.001(2)$ & $0.003(1)$ & $-0.024(2)$ \\
\hline$C(16)$ & $4 e$ & $0.8855(2)$ & $0.0117(6)$ & $0.6501(1)$ & $0.065(2)$ & $0.027(2)$ & $0.026(1)$ & $0.006(1)$ & $0.017(1)$ & $-0.008(1)$ \\
\hline$C(17)$ & $4 e$ & $0.8114(2)$ & $-0.1225(6)$ & $0.6462(1)$ & $0.032(2)$ & $0.071(3)$ & $0.026(1)$ & $0.014(2)$ & $0.012(1)$ & $-0.012(1)$ \\
\hline$C(18)$ & $4 e$ & $0.8016(1)$ & $-0.2875(4)$ & $0.50824(9)$ & $0.028(1)$ & $0.021(2)$ & $0.015(1)$ & $0.001(1)$ & $0.0049(9)$ & $-0.0024(9)$ \\
\hline$C(19)$ & $4 e$ & $0.8340(2)$ & $-0.5052(5)$ & $0.5308(1)$ & $0.035(1)$ & $0.022(2)$ & $0.023(1)$ & $0.000(1)$ & $0.009(1)$ & $-0.005(1)$ \\
\hline$C(20)$ & $4 e$ & $0.9269(2)$ & $-0.4963(5)$ & $0.5487(1)$ & $0.035(1)$ & $0.032(2)$ & $0.026(1)$ & $0.011(1)$ & $0.011(1)$ & $-0.005(1)$ \\
\hline$C(21)$ & $4 e$ & $0.9522(2)$ & $-0.2776(5)$ & $0.5376(1)$ & $0.028(1)$ & $0.040(2)$ & $0.025(1)$ & $0.001(1)$ & $0.013(1)$ & $-0.004(1)$ \\
\hline$C(22)$ & $4 e$ & $0.8754(2)$ & $-0.1448(5)$ & $0.5127(1)$ & $0.031(1)$ & $0.027(2)$ & $0.016(1)$ & $-0.001(1)$ & $0.0096(9)$ & $0.002(1)$ \\
\hline$C(23)$ & $4 e$ & $0.7078(2)$ & $-0.2233(5)$ & $0.4894(1)$ & $0.030(1)$ & $0.026(2)$ & $0.018(1)$ & $-0.002(1)$ & $0.0018(9)$ & $0.001(1)$ \\
\hline$C(24)$ & $4 e$ & $0.6065(2)$ & $0.0773(6)$ & $0.4653(2)$ & $0.027(1)$ & $0.035(2)$ & $0.051(2)$ & $0.005(1)$ & $0.002(1)$ & $0.002(2)$ \\
\hline
\end{tabular}

Acknowledgment. We thank the Fonds der Chemischen Industrie for generous support.

\section{References}

1. Benkeser, R. A.; Goggin, G.: A Route to Monosubstituted Ferrocene Compounds. J. Am. Chem. Soc. 76 (1954) 4025-4026.

2. Gmelin Handbuch der Anorganischen Chemie, Eisenorganische Verbindungen Bd. 50, Teil A, Ferrocen 3 (1978).

3. Wolf, L.; Hennig, H.: Ferrocensubstituierte 1,3-Diketone. Z. Chem. 12 (1963) 469-470.

4. Beyer, L.; Richter, R.; Seidelmann, D.: Ferrocensubstituierte 1,3-bidentate Liganden und ihre heteronuklearen Übergangsmetallchelate. J. Prakt. Chem. 341 (1999) 704-726.

5. Woisetschläger, O. E.; Geisbauer, A.; Polbom, K.; Sünkel, K.; Beck, W.: Spacer verbrickte Bis-, Tris- und Tetrakis(ferrocenyl)-1,3-Diketone. Z. Anorg. Allg. Chem. 625 (1999) 2164-2168.

6. Fischer, D. W.: Crystallographic data for some ferrocene derivatives. Acta Crystallogr. 17 (1964) 619.
7. Cotton, F. A.; Reid, A. H.: Solid-State Structure of Ferrocenecarboxylic Acid. Acta Crystallogr. C41 (1985) 686-688.

8. Takusagawa, F.; Koetzle, T. F.: The Crystal and Molecular Structure of 1,1'-Ferrocenedicarboxylic Acid. Acta Crystallogr. B35 (1979) 2888-2896.

9. Altomare, A.; Burla, M. C.; Camalli, M.; Cascarano, G. L., Giacovazzo, C.; Guagliardi, A., Moliterni, A. G. G.; Polidori, G.; Spagna, R.: SIR97: a new tool for crystal structure determination and refinement. J. Appl. Crystallogr. 32 (1999) 115-119.

10. Sheldrick, G. M.: SHELXL-97. Program for the Refinement of Crystal Structures. University of Göttingen, Germany 1997.

11. Burnett, M. N.; Johnson, C. K. : ORTEP-III: Oak Ridge Thermal Ellipsoid Plot Program for Crystal Structure Illustrations, Oak Ridge National Laboratory Report ORNL-6895, USA 1996. Windows version used (L. J. Farrugia, University Glasgow). 